\title{
A Simple and Efficient Scan Correlation Algorithm for Sea Surveillance Radar
}

\author{
Byung-Doo Kim ${ }^{1}$, Seung-Hee $\mathrm{Oh}^{1}$, and Byung-Gil Lee ${ }^{1}$ \\ Convergence Security Research Section, Electronics and Telecommunications \\ Research Institute, Daejeon, Korea \\ (bdkim, seunghee, bglee)@etri.re.kr
}

\begin{abstract}
In most sea surveillance radars based on non-coherent integration, the scan integration which improves signal to noise ratio and mitigates unwanted echo with a small correlation time such as sea clutter is widely used. The problems of the conventional scan integration are: (a) the target trail is generated through integrating prior scan and current scan; (b) it is difficult to detect a small and fast moving target because of the attenuated small target echo caused by scan integration. In this paper, the conventional scan integration algorithm is analyzed in order to overcome the above problems, and then a simple and efficient scan correlation algorithm is proposed to remove the target trail. The proposed scan correlation algorithm uses the difference the current and the prior CFAR processed scan video. Compared to the conventional scan correlation algorithm, the proposed method is very simple and efficiently removes the target trail. The computer simulation results show that the proposed algorithm can extract a fast moving target without a target trail.
\end{abstract}

Keywords: Sea Clutter, Scan Integration, Constant False Alarm Rate, Target Trail Removal, Scan Correlation, Sea Surveillance Radar

\section{Introduction}

Radar unfortunately receives not only desired target echo but also unwanted echo caused by the natural environment. Clutter is the term used to denote unwanted echoes and can be classified into rain, cloud, ground, sea, and others [1-2]. It makes difficult the detection of wanted targets. Especially, sea clutter depending on the shape of the sea surface is not so easily canceled as ground clutter since the former is moving and fluctuating while the latter is almost fixed. Sea clutter becomes a more serious problem when small, slowly moving boats have to be detected within sea waves.

A large proportion of radars use only the envelope of the received signal in their processing. Since they do not use the signal phase, these systems can be non-coherent from pulse to pulse, and in consequence can use simple transmitters such as pulsed magnetrons and their receivers may employ a logarithmic detector that provides wide "dynamic range" and facilitates the analog calculation of products, ratios, and powers. The envelope of sea clutter after logarithmic detector is distributed roughly according to a Rayleigh probability distribution [3-5]. In most sea surveillance radars with non-coherent integration, Scan Integration (SI) algorithm is used to extract stationary and slow moving targets with a small signal to noise ratio and suppress unwanted echo with small correlation time. Since the scan rate of sea surveillance radar is about 3 to 30 revolutions per minute, echoes of sea clutter at the identical position from two consecutive scans are obviously uncorrelated to each other [6]. The problems of conventional SI are that a target trail is generated by integration of the current scan and prior scans for fast target with high Radar Cross Section (RCS), and the detection of a small, fast target is difficult due to attenuated small target echo. For the purpose of target trail removal, the conventional 
Scan Integration/Correlation (SIC) algorithm comprising signal weighting and signal selection is used. In the conventional Scan Correlation (SC) algorithm, two thresholds are required, derived from the clutter level [7]. For each resolution cell, if prior integrated scan amplitude and current scan amplitude concurrently exceed the two thresholds, the prior integrated scan amplitude is fed to the next processing stage; otherwise a zero is sent. Generally, this conventional algorithm can only detect small, stationary, and slowly moving targets, and has more complexity.

In this paper, the problems of the conventional scan integration are analyzed by using a series analysis and then, a simple and efficient scan correlation algorithm is proposed. The proposed scan correlation algorithm uses the difference the current and the prior constant false alarm rate (CFAR) processed scan video. Compared to the conventional scan correlation algorithm using three thresholds, the proposed method is very simple because it operates with only one threshold. Also, the proposed method efficiently removes the target trail.

\section{Scan Integration}

\subsection{Radar Signal Processing}

The signal processing of a sea surveillance radar system suffers from sea clutter, receiver thermal noise, etc. There are so many signal processing techniques to suppress the unwanted echoes and detect target echoes.

The reflected echo is represented with the consideration of two cases in which the input has the target echo, the clutter echo and the receiver noise and only the clutter echo and the noise [8]. Hence, the radar video can be written as:

$$
r_{n}(t)= \begin{cases}s_{n}(t)+c_{n}(t)+v_{n}(t) & t=t_{n} \\ c_{n}(t)+v_{n}(t) & t \neq t_{n}\end{cases}
$$

where $n$ is the scan index, $t_{n}$ is the elapsed time corresponding to the target range, $s_{n}(t)$ is the target echo, $c_{n}(t)$ is the clutter that assumed a short time correlated and normally distributed process. $v_{n}(t)$ is the receiver noise that assumed a white Gaussian noise.

The radar video is sampled by an Analog to Digital (A/D) converter for digital signal processing. A/D converted video data are stored during several Pulse Repetition Intervals (PRIs), and those are processed by a pulse to pulse integration algorithm to obtain improved Signal to Noise Ratio (SNR). This process is called the non-coherent integration. The pulse integrated video can be written as:

$$
a_{n}[k] \cong \begin{cases}h_{1, n}[k] \quad k=p_{n} \\ h_{0, n}[k] \quad k \neq p_{n}\end{cases}
$$

where $h_{1, n}[k]$ is the video with the target echo at the $n$-th scan, $h_{0, n}[k]$ is the video without the target echo at the $n$-th scan, $k$ is the range cell index and $p_{n}$ is the range cell index with respect to the target range.

\subsection{Conventional Scan Integration}

The conventional scan integration is performed to allow increasing Signal to Clutter Ratio (SCR) for the sea clutter with short time correlation. Generally, the sea clutter spike occurs at a random range cell for consecutive scans. By recursive integration of several 
scans, the clutter level is reduced while stationary target amplitude remains. In this way, small stationary targets emerge from the clutter.

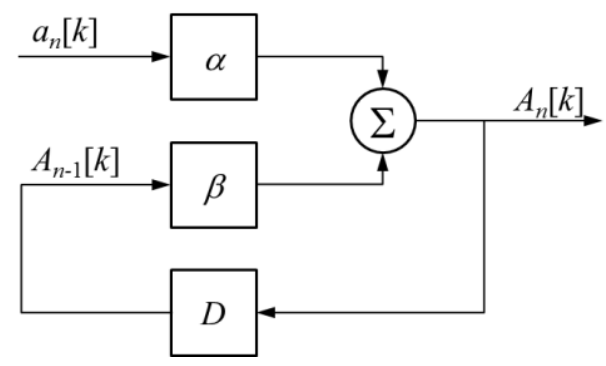

Figure 2. Conventional Scan Integration Algorithm

The conventional scan integration can be expressed by recursive equation given by:

$$
A_{n}[k]=\alpha \cdot A_{n-1}[k]+\beta \cdot a_{n}[k]
$$

where $A_{n-1}[k], A_{n}[k]$ is the integrated video up to the $(n-1)$-th and the $n$-th scan, respectively. $a_{n}[k]$ is the current scan video at the $n$-th scan and $\alpha, \beta$ is the weighting factor for the priori integrated video and the current video, respectively.

The recursive equation in (3) can be rewritten as follows:

$$
A_{n}[k]=\beta \sum_{m=0}^{n-1} \alpha^{m} a_{n-m}[k]
$$

where

$$
a_{n-m}[k]= \begin{cases}h_{1, n-m}[k] & k=p_{n} \\ h_{0, n-m}[k] & k \neq p_{n}\end{cases}
$$

Substituting (5) into (4) results in:

$$
A_{n}[k]= \begin{cases}\beta \sum_{m=0}^{n-1} \alpha^{m} \cdot h_{1, n-m}[k] & k=p_{n} \\ \beta \sum_{m=0}^{n-1} \alpha^{m} \cdot h_{0, n-m}[k] & k \neq p_{n}\end{cases}
$$

\subsection{Analysis of Scan Integration}

In the case of a stationary target, the cell index of the target range at the current scan is assumed to be almost identical with those at the prior scans. In addition, if the target fluctuation is according to the Swerling model 0 (i.e., constant RCS), the amplitude of the sampled target echo is analogous with those at the prior scans. Therefore, the following assumption can be made:

$$
h_{1,1}\left[p_{1}\right] \cong h_{1,2}\left[p_{2}\right] \cong \cdots \cong h_{1, n}\left[p_{n}\right]
$$

From the above assumption, the expectation of (6) is derived by using the geometric series and the constraint the weighting factor of SI, which can be written as: 


$$
E\left\{A_{n}[k]\right\}= \begin{cases}\bar{h}_{1}[k] & k=p_{n} \\ \bar{h}_{0}[k] & k \neq p_{n}\end{cases}
$$

where

$$
E\left\{\beta \cdot \sum_{m=0}^{n-1} \alpha^{m} h_{1, n-m}[k]\right\} \cong \bar{h}_{1}[k]
$$

The expectation of integrated scan without target echo after SI can be obtained by same approach. As one can see, the radar video with target echo and non-target echo are attenuated to mean level. Generally, because the amplitude of the radar video with target echo is greater than the amplitude of the radar video with non-target echo, the CFAR processing can detect the target. In the case of a fast moving target, its cell index of the target range at the current scan can be assumed to be different from those at the prior scans. From these factors, the following assumption can be made:

$$
p_{1} \neq p_{2} \neq \cdots \neq p_{n}
$$

According to (10), the expectation of (6) can be derived as the following equation.

$$
E\left\{A_{n}[k]\right\}= \begin{cases}\beta h_{1, n}[k]+\alpha \Delta h[k] & k=p_{n} \\ \bar{h}_{0}[k] & k \neq p_{n}\end{cases}
$$

where

$$
\begin{gathered}
\Delta h[k]=\beta h_{1, n-1}[k]+\cdots+\beta \alpha^{n-2} h_{1,1}[k] \\
E\left\{\beta \sum_{m=0}^{n-1} \alpha^{m} h_{1, n-m}[k]\right\}=\beta h_{1, n}[k]+\beta \alpha h_{1, n-1}[k]+\beta \alpha^{n-1} h_{1,1}[k]
\end{gathered}
$$

As one can see from (11), not only the video amplitude contained target echo at the current scan is attenuated by signal weighting, $\beta$ but the target trail denoted by $\alpha \Delta h$ is also generated as a side effect of SI, and $\Delta h$ is described as the result of SI until prior scan by (12). The averaged video with non-target echo after SI can be detected as a new target by CFAR processing if the amplitude of the target trail is greater than the averaged video with non-target echo.

\section{Scan Correlation}

The objective of the CFAR design is to provide a detection threshold that is relatively immune to variations of the background clutter-plus-noise level and to allow target detection with a CFAR [9]. A great variety of CFAR processing algorithms [10-12] are widely used including the well-known Cell Averaging (CA) CFAR detector which is used in this paper.

In this approach, the proposed scan correlation algorithm decides whether the detected target by CFAR processing is the real target or the target trail caused by scan integration. If the difference of a current CFAR video and the prior CFAR video multiplied by $\alpha$ (weighting factor of prior scan in scan integration) is greater than a decision threshold, then the detected target is determined as a real target. Otherwise the detected target is regarded as a target trail. The radar signal processing structure with the proposed scan correlation algorithm can be configured as follows: 


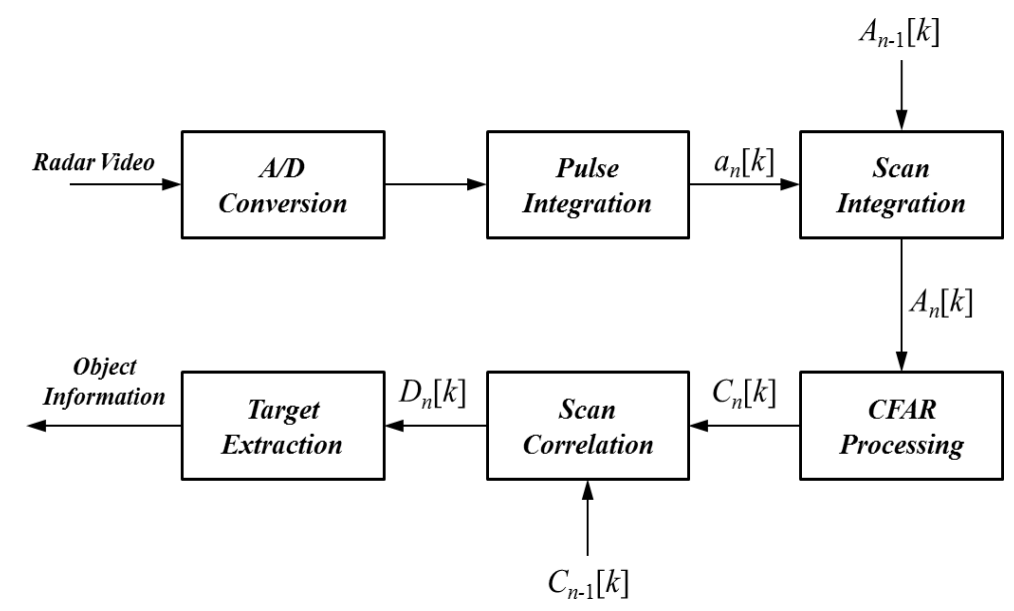

Figure 3. Proposed Signal Processing Structure with Scan Correlation Algorithm

From (8), that is to say, in the case of the stationary target, the CFAR video can be denoted as follows:

$$
C_{n}[k]= \begin{cases}\overline{h_{1}}[k] & k=p_{n} \\ 0 & k \neq p_{n}\end{cases}
$$

The difference between the current CFAR video and the prior CFAR video multiplied by $\alpha$ is described as follows:

$$
C_{n}[k]-\alpha \cdot C_{n-1}[k]= \begin{cases}\beta \cdot \bar{h}_{1, n}[k] & k=p_{n} \\ 0 & k \neq p_{n}\end{cases}
$$

Also, from (11) and (12), the CFAR video of the fast moving target can be denoted as follows:

$$
C_{n}[k]= \begin{cases}\beta \bar{h}_{1, n}[k]+\beta \alpha \bar{h}_{1, n-1}[k]+\cdots+\beta \alpha \bar{h}_{1, n-j}[k] & k=p_{n} \\ 0 & k \neq p_{n}\end{cases}
$$

where $j$ is the scan index satisfied the condition, $\beta \alpha^{j} \bar{h}_{1, n-j}\left[p_{n-j}\right]>V_{T H}$ and $V_{T H}$ is the CFAR threshold. As one can see, the target trail is generated by the fast moving target from (16). This target trail has to be removed because it is a virtual target due to the scan integration. The difference between the current CFAR video and the prior CFAR video multiplied by $\alpha$ is described as follows:

$$
C_{n}[k]-\alpha \cdot C_{n-1}[k]= \begin{cases}\beta \cdot \bar{h}_{1, n}[k]-\beta \alpha^{j+1} \bar{h}_{1, n-j-1}[k] & k=p_{n} \\ 0 & k \neq p_{n}\end{cases}
$$

Since the second term, $\beta \alpha^{j+1} \bar{h}_{1, n-j-1}[k]$ is very small, therefore the above equation can be simplified as follows: 


$$
C_{n}[k]-\alpha \cdot C_{n-1}[k] \cong \begin{cases}\beta \cdot \bar{h}_{1}[k] & k=p_{n} \\ 0 & k \neq p_{n}\end{cases}
$$

From (15) and (18), the output of the scan correlation algorithm can be expressed as follows:

$$
D_{n}[k]= \begin{cases}C_{n}[k] & \delta \leq C_{n}[k]-\alpha \cdot C_{n-1}[k] \\ 0 & \delta>C_{n}[k]-\alpha \cdot C_{n-1}[k]\end{cases}
$$

The proposed scan correlation algorithm can be drawn as follows:

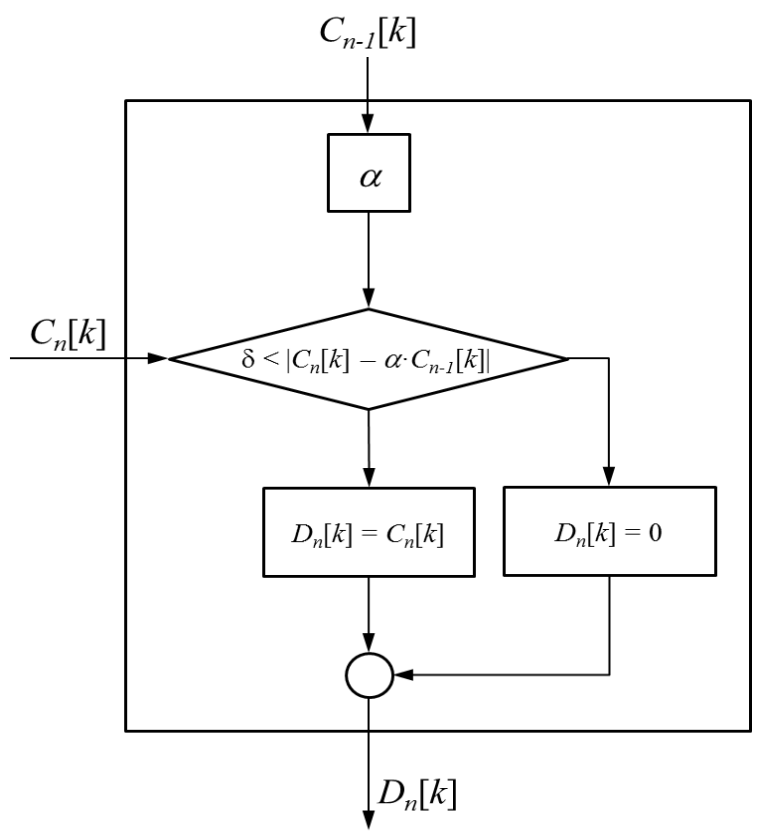

Figure 4. Proposed Scan Correlation Algorithm

For the implementation of the proposed scan correlation algorithm, the decision threshold should be set considering the fluctuation of target echo and variation of sea clutter.

\section{Computer simulation}

A computer simulation is performed to evaluate the proposed scan correlation algorithm's performance of detecting a small and fast moving target without target trail effect.

The simulated radar video has five targets, sea clutter and receiver thermal noise. Five target echoes are configured two stationary targets and three moving targets with different speeds that are 30,60, 90 knots of radial velocity, respectively. Also, sea clutter is generated utilizing Morchin's sea clutter model [13] with Rayleigh distribution at sea state 3 . The simulated radar video is sampled by 8 bits $\mathrm{A} / \mathrm{D}$ converter. According to the proposed signal processing structure, sampled A-scope videos are fed into the pulse integration step. In this paper, the pulse integration is performed by Censored Video Integration (CVI) [14] algorithm with 8 pulses. The CVI follows Ritcey's estimator which combines sorting and censoring with an averaging of the remaining samples with a non- 
uniform weighting law and is also immune to spikes, yet suffers a smaller loss than the binary integrator.

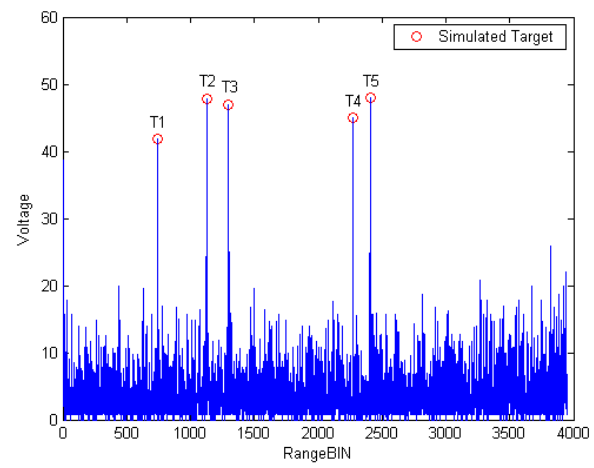

Figure 5. Pulse Integrated A-scope Video

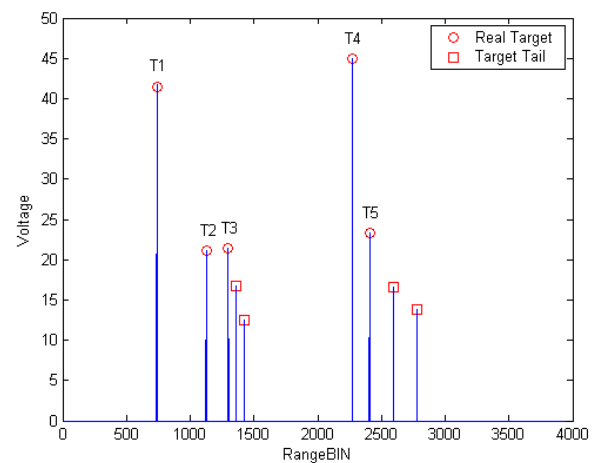

Figure 7. CFAR Processed A-scope Video

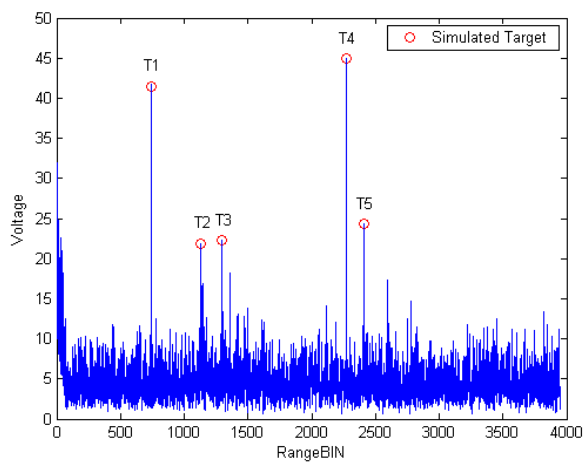

Figure 6. Scan Integrated A-scope Video

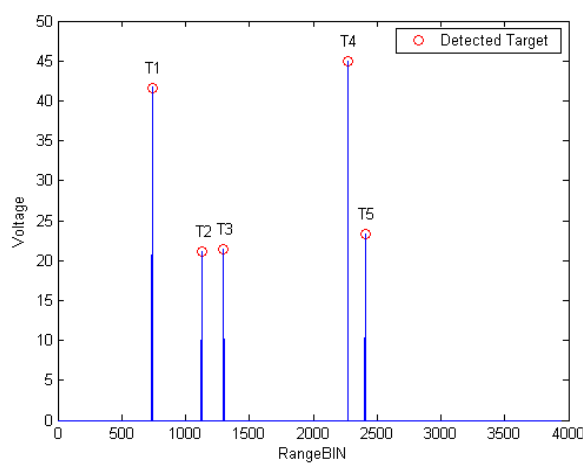

Figure 8. Scan Correlated A-scope Video

The results of the pulse integration and the scan integration are depicted in Figure 5 and Figure 6, respectively. After conventional SI, it is shown that sea clutter is suppressed but target trails are generated. In this paper, the Cell Averaging (CA) CFAR algorithm [15] is employed. In Figure 7, the target trails generated by T3 and T5 are regarded as new targets by CFAR processing. These targets are the virtual targets of T3 and T5 and should be removed using a scan correlation algorithm. The result of the proposed scan correlation algorithm is depicted in Figure 8. Only the real target echo is detected without the target trails.

\section{Conclusion}

In this study, the conventional scan integration has been analyzed by using the geometric series and the novel scan correlation algorithm using the difference of the consecutive CFAR processed video has been proposed to detect a small and fast moving target through removing its trail. The computer simulation is carried out to evaluate the performance of the proposed scan correlation algorithm. The simulation results show that the proposed algorithm is very effective to detect a small and fast moving target without the target trail. Additionally, the position accuracy which can be extracted from radar image by the centroid method [16] can be improved by the removal of the target trails. 


\section{Acknowledgements}

This work was supported by ETRI through Maritime Safety \& Maritime Traffic Management R\&D Program of the MOF/KIMST (2009403, Development of Next Generation VTS for Maritime Safety).

This paper is a revised and expanded version of a paper entitled "A study on probabilistic stranding warning for maritime safety control system" presented at Saipan on July 17-20, 2014(ITCS 2014).

\section{References}

[1] M. I. Skolnik, Introduction to Radar Systems 3rd ed., McGraw-Hill, New York (2001).

[2] D. K. Barton, Modern Radar System Analysis. Artech House, Massachusetts (1988)

[3] K. D. Ward, C. J. Baker and S. Watts, "Maritime surveillance radar Part 1: Radar scattering from the ocean surface," IEE Proceedings, Vol. 137, pp.51-62, (1990)

[4] B. A. Green, "Radar detection probability with logarithmic detectors," IRE Transactions on Information Theory, Vol. 4, pp.50-52 (1958).

[5] J. Croney, "Clutter on radar displays-reduction by use of logarithmic receivers," Wireless Engineer, Vol. 33, pp.83-95 (1956).

[6] H. C. Shyu, Y. S. Sun, and W. H. Shen, "The Analysis of Scan-to-Scan Integration Techniques for Sea Clutter," Proc. of IEEE National Radar Conference, Atlanta, GA, USA, pp.228-233 (1994)

[7] F. X. Hofele, "Scan to Scan Integration Correlation for the Detection of Small Fast Targets," Proc. of CIE International Conference on Radar, Beijing, China, pp.380-384 (2001)

[8] L. J. Spafford, "Optimum radar signal processing in clutter," IEEE Transactions on Information Theory, Vol. 14, 734-743 (1968)

[9] C. J. Kim and H. J. Lee, "Performance Analysis of the Clutter Map CFAR Detector with Noncoherent Integration,” ETRI Journal, Vol. 15, pp.1-9, (1993)

[10] H. R. Park, Y. K. Kwag, and H. Wang, "An Efficient Adaptive Polarimetric Processor with an Embedded CFAR,” ETRI Journal, Vol. 25, pp.171-178, (2003)

[11] P. Radu, K. Sirlantzis, W. G. J. Howells, F. Deravi, and S. Hoque, "Information Fusion for Unconstrained Iris Recognition," International Journal of Hybrid Information Technology, Vol. 4, pp.112, October, (2011)

[12] S. Tarar and E. Kumar, "Fingerprint Image Enhancement: Iterative Fast Fourier Transform Algorithm and Performance Evaluation,” International Journal of Hybrid Information Technology, Vol. 6, pp.11-20, July, (2013)

[13] B. Edde, Radar Principles, Technology, Applications. Prentice Hall, (1993)

[14] N. Levanon, "Censored Video Integration in Radar Detection," IEEE International Radar Conference, Arlington, VA, USA, pp.511-513 (1990)

[15] G. V. Weinberg, Estimation of False Alarm Probabilities in Cell Averaging Constant False Alarm Rate Detectors via Monte Carlo Methods, DSTO-TR-1624, System Sciences Lab., Edinburgh, Australia, (2004)

[16] G. R. Painchaud, A Survey of Automatic Detection and Plot Extraction Techniques for Surveillance Radar, CRC Report No. 1334, Communications Research Center, Ottawa, Canada, (1980).

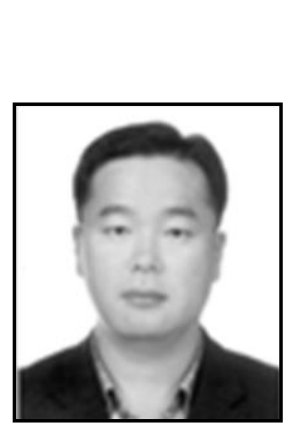

\section{Authors}

Byung-Doo Kim received the M.S. and Ph.D. degrees from the Department of Electronics Engineering from Ajou University, Korea, in 1998 and 2007, respectively. In 2002, he was with MteQ Systems, Korea, where he worked on the research of signal processing and tracking algorithm of sea surveillance radar systems. Since 2004, he has been with Electronics and Telecommunications Research Institute (ETRI), Korea, as a Senior Researcher. His research interests include multi-target tracking, signal processing of radar system, and navigation algorithm based on GNSS for LBS. 


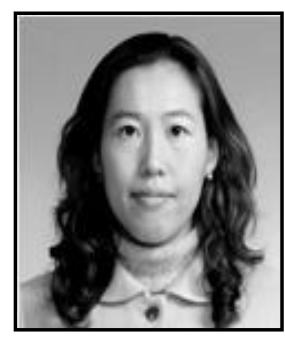

Seung-Hee Oh received her BS degree in Computer Science from Chonbuk National University, Korea, in year 1999. She received her MS degree in Computer Science and Engineering from Ewha Womans University, Korea, in 2001. Since 2001, she has been a researcher in Electronics and Telecommunications Research Institute (ETRI), Korea. Her research interests include information security, network security, security management, air traffic control, vessel traffic control, etc.

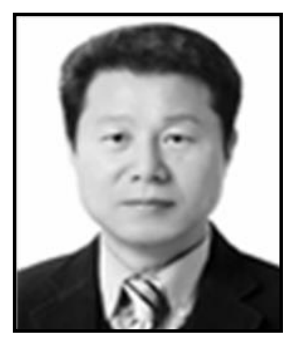

Byung-Gil Lee received his $\mathrm{BS}$, MS and $\mathrm{Ph} . \mathrm{D}$ degree in electrical engineering from the Kyungpook National University, Daegu, Rep. of Korea, in 1999, 2001 and 2009, respectively. In 2001, he joined the research member of Electronics and Telecommunications Research Institute (ETRI) in Korea and is currently a project leader of maritime safety research project. His current research interests in wired/wireless network security and ICT based maritime safety. 
International Journal of Control and Automation

Vol. 7, No. 11 (2014) 